\title{
High VCAM-I Predicts Poor Prognosis and is Associated with Chemotherapy Resistance in Nasopharyngeal Carcinoma
}

This article was published in the following Dove Press journal: OncoTargets and Therapy

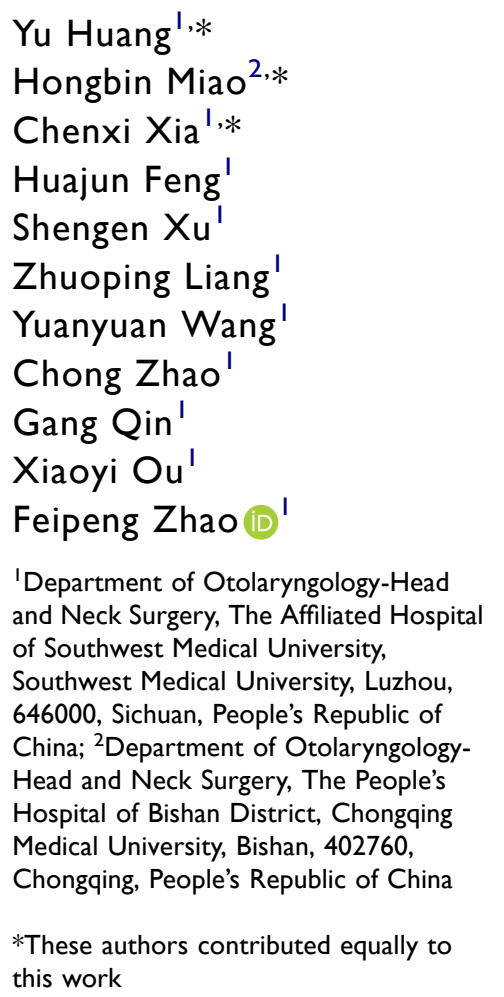
this work
Purpose: Nasopharyngeal carcinoma (NPC) is a malignant tumor endemic in southern China and Southeast Asia with a poor prognosis. Vascular cell adhesion protein 1 (VCAM-1) is highly expressed in NPC; however, it is unclear whether VCAM-1 is correlated with chemotherapy resistance and prognosis in NPC.

Patients and Methods: To further explore the role of VCAM-1 in chemotherapy resistance and prognosis in NPC, we examined the expression of VCAM-1, the sensitivity of chemotherapy drugs, and clinical follow-up data from 73 patients with NPC. Then, the results of VCAM-1 expression were analyzed in response to chemotherapy drugs, progression-free survival (PFS), and overall survival (OS).

Results: The expression of VCAM-1 protein in NPC was significantly higher than that in chronic inflammatory tissue. No significant differences in the expression of VCAM-1 among gender, age, pathologic classification, tumor classification, lymph node status, metastasis status, and overall clinical stage were found. The periods of PFS and OS in patients with high VCAM-1 expression were significantly shorter than those in patients with low VCAM-1 expression. The sensitivity rates of NPC to eight chemotherapy drugs were different; carboplatin and docetaxel showed the highest chemotherapy sensitivity and resistance rates, respectively. The resistance rates to paclitaxel were different between the patients with high VCAM-1 expression and those with low VCAM-1 expression.

Conclusion: Our data indicated that VCAM-1 was highly expressed in NPC. Patients with high VCAM-1 expression were more prone to shorter periods of PFS and OS. VCAM-1 could be a prognostic marker of NPC patients. The detection of VCAM-1 expression in NPC may be valuable for chemotherapy drug evaluation and management of patients with NPC in the clinic.

Keywords: nasopharyngeal carcinoma, VCAM-1, prognosis, chemotherapy resistance

\section{Introduction}

Nasopharyngeal carcinoma (NPC) is a malignant tumor of the head and neck that originates in the mucosa of the nasopharyngeal region, which has a high rate of incidence in Southern China and Southeast Asia. It is clinically characterized by poor differentiation and a strong tendency for early metastasis, leading to a poor prognosis. ${ }^{1}$ Radiotherapy is the primary choice of treatment for patients with early NPC. Chemotherapy and even surgery are indispensable treatment strategies for highly differentiated cancers, advanced stage cancers, and recurrent cancers after radiotherapy in NPC. Although chemotherapy combined with radiotherapy has
Zhao

Department of Otolaryngology-Head and Neck Surgery, The Affiliated Hospital of Southwest Medical University, No. 25 Taiping Street, Luzhou, 646000, Sichuan Province, People's Republic of China Tel +86-830-3 I6564l

Fax +86-830-2392753

Email1603683884@qq.com;

379771630@qq.com 
significantly improved the survival rate and quality of life in patients with locally advanced NPC, the five-year survival rate of patients with stage 3-4 NPC is approximately $70 \%{ }^{2,3}$ The major reasons for treatment failure are resistance to radiotherapy and chemotherapy drugs, local recurrence, and distant metastasis. At present, miR-34c, ${ }^{4}$ miR205-5p, ${ }^{5}$ TRIM1 $1,{ }^{6} \mathrm{CDKs}^{7}$ and melatonin ${ }^{8}$ are related to chemoresistance of NPC, which affects the prognosis of patients with NPC. Given that the specific mechanism of chemoresistance of NPC remains unclear, exploring novel molecular targets for chemotherapy drug resistance in NPC would benefit the effectiveness of clinical treatment and prognosis of patients.

Vascular cell adhesion protein 1 (VCAM-1, CD106), first identified in 1989 , is a $90-\mathrm{kDa}$ glycoprotein expressed on the endothelial cell surface. ${ }^{9}$ The production of proinflammatory cytokines, including TNF $\alpha$, ROS, toll-like receptor agonists, or shear stress induces VCAM-1 expression. ${ }^{10}$ Under certain chronic inflammatory stimuli, VCAM-1 is also expressed on the surface of other cells, including tissue macrophages, dendritic cells, bone marrow fibroblasts, myoblasts, oocytes, Kupffer cells, and cancer cells. ${ }^{11}$ As a cell adhesion protein, VCAM-1 plays an important role in inflammation and immunological disorders. ${ }^{12}$ VCAM-1 is highly expressed in breast cancer ${ }^{13,14}$ and gastric cancer, ${ }^{15}$ and is related to tumor angiogenesis and metastasis. Previous studies have shown that patients with increased expression of VCAM-1 in colorectal cancer, ${ }^{16}$ lung cancer, ${ }^{17}$ and ovarian cancer $^{18}$ are more prone to distant metastasis and poor prognosis. VCAM-1 could thus be considered as a potential target for anti-vascular and antimetastatic cancer therapy. ${ }^{19}$ VCAM-1 is associated with the chemoresistance of gastric cancer. ${ }^{20}$ High expression of VCAM-1 in breast cancer can confer chemoresistance to adriamycin or cisplatin. ${ }^{21}$ Inhibition of VCAM-1 in acute myeloid leukemia (AML) enhances the sensitivity to chemotherapy drugs. ${ }^{22}$ Recent studies have revealed that VCAM-1 is highly expressed in NPC and is related to the angiogenesis of NPC. ${ }^{23,24}$ However, it is unclear whether the high expression of VCAM-1 is related to chemotherapy resistance and prognosis in NPC.

To further explore the role of VCAM-1 in chemotherapy resistance and prognosis, we examined the expression of VCAM-1, the sensitivity of chemotherapy drugs, and clinical follow-up data in 73 patients with NPC. The results of VCAM-1 expression were then analyzed in response to chemotherapy drugs, progression-free survival (PFS), and overall survival (OS).

\section{Patients and Methods}

\section{Clinical Data}

During the nasopharyngeal biopsy operation, the tumor samples of every NPC patient in this study were divided into three parts, one for pathological diagnosis, one for tumor chemosensitivity testing, and one for paraffin tissue specimens. Seventy-three fresh cancer tissues and corresponding paraffin tissue specimens from newly-diagnosed NPC patients were collected before treatment at the Affiliated Hospital of Southwest Medical University from March 2014 to August 2016, including 49 males and 24 females, aged 21-77 years, with a median age of 48 years. All patients with NPC were confirmed by pathological examinations and staged according to the seventh edition of the American Joint Committee on Cancer (AJCC). Thirty patients (and their tissue specimens) diagnosed with chronic nasopharyngeal inflammation at the Affiliated Hospital of Southwest Medical University during the same period were taken as the control group, including 15 males and 15 females, aged 17-75 years, with a median age of 47 years. The follow-up methods were mainly outpatient review or telephone return visits during the median observation of 30 months (range, 9-45 months).

This study was approved by the Ethics Committee of the Affiliated Hospital of Southwest Medical University (NO.K2016050), which was conducted under the Declaration of Helsinki. And informed consent was obtained from all patients before treatment. If the patient was under 18 years of age, the informed consent was signed by a parent or legal guardian of the patient.

\section{Immunohistochemistry and Statistical Analysis}

Immunohistochemistry (IHC) was performed to analyze the expression of VCAM-1 protein in nasopharyngeal inflammation and NPC tissues by the Envision two-step IHC method. Briefly, $4 \mu \mathrm{m}$ dewaxed sections were processed with EDTA $(\mathrm{pH}=9.0)$ for high-pressure antigen retrieval, incubated in $\mathrm{H}_{2} \mathrm{O}_{2}(3 \%)$ for 10 min to eliminate endogenous peroxidase activity, washed three times with PBS buffer, blocked in $1 \%$ goat serum albumin for $1 \mathrm{~h}$, incubated with VCAM-1 antibody (rabbit anti-human VCAM-1 antibody, 1: 100 dilution; \#12367; Cell Signaling Technology, USA) overnight at $4^{\circ} \mathrm{C}$, and then incubated with secondary antibody (Kit-9921; MXB Biotechnologies, Fuzhou, China) for $30 \mathrm{~min}$ at $25^{\circ} \mathrm{C}$. 
Next, sections were stained using the 3,3'diaminobenzidine staining fluid detection kit (DAB-0031; MXB Biotechnologies, Fuzhou, China). Then, the sections were counterstained with hematoxylin for $2 \mathrm{~min}$, dehydrated, and mounted. All procedures were carried out in strict accordance with the reagent instructions. Stained sections were observed and evaluated under a microscope (BX60, Olympus, Japan). Three images of each section were obtained in different positions under a high-power field $(400 \mathrm{x})$ of the microscope. Then, the MOD value of VCAM-1 protein expression was analyzed using Image-Pro Plus 6.0 image analysis software.

\section{In vitro ATP-TCA}

Tumor chemosensitivity was assessed with primary cell cultures using the ATP-based tumor chemosensitivity assay (ATP-TCA) kit (Kit-2005-3070035; Jinzijing Biotechnologies, Beijing, China) according to the manufacturer's instructions. In all cases, ATP-TCA data was obtained as previously published. ${ }^{25}$ Briefly, fresh cancer tissues $\left(0.3-0.5 \mathrm{~cm}^{3}\right)$ were obtained from biopsies and cut into pieces of $1 \mathrm{~mm}^{3}$. Tumor cells were isolated by trypsin dissociation to obtain a single-cell suspension. After cell counting, $2-4 \times 10^{4}$ cells were seeded into each well of a 96-well polypropylene microplate. Subsequently, cells were treated with a gradient of five dilutions (12.5, 25, 50,100 , and $200 \%$ ) of each chemotherapy drug in duplicates. Two controls were included in each plate: a no-drug control consisting of media only and a maximum inhibitor control which kills all cells present. Cells were cultured for five days at $37^{\circ} \mathrm{C}, 5 \% \mathrm{CO}_{2}$, and saturated humidity. Each well was treated with $50 \mu \mathrm{L}$ ATP extraction reagent. Then, $50 \mu \mathrm{L}$ of the mixed solution from each well was added to the corresponding wells in a blank 96-well polypropylene microplate, followed by the addition of luciferin-luciferase assay reagents. The light output was measured using a microplate luminometer (Hamamatsu, Beijing, China) and analyzed with custom software to obtain both numerical and graphical results, which included the tumor growth inhibition rate at each concentration, the IC50 and IC90 values, and the inhibition curve of each chemotherapy drug.

Eight chemotherapy drugs used in this study were fluorouracil (Hengrui Medicine, China), cisplatin (Qilu Medicine, China), carboplatin (Qilu Medicine, China), oxaliplatin (Hengrui Medicine, China), paclitaxel (Beijing Union Pharmaceutical Factory, China), docetaxel (Hansoh Pharma, China), gemcitabine (Hansoh Pharma, China), and cyclophosphamide (Hengrui Medicine, China). The evaluation criteria for chemotherapeutic drug sensitivity ${ }^{25}$ were as follows: high sensitivity (IC50 <25\% PPC and IC90 <100\% PPC), moderate sensitivity (IC50 $<25 \% \quad$ PPC and IC90>100\% PPC), mild sensitivity (IC50 >25\% PPC and IC90<100\% PPC), and resistance (IC50>25\% PPC and IC90>100\% PPC). IC90 is the peak plasma concentration of the chemotherapy drug which inhibited tumor growth by $90 \%$. IC50 is the peak plasma concentration of the chemotherapy drug which inhibited tumor growth by $50 \%$. PPC is the peak plasma concentration of the chemotherapy drug corresponding to a certain clinical dose.

\section{Statistical Analyses}

All statistical analyses were performed using SPSS software (IBM SPSS Statistics, version 19.0; Chicago, IL, USA). Differences in the measured data were compared using the $t$-test. Count data were compared using the chisquared $\left(\chi^{2}\right)$ test. Survival outcomes were analyzed using the Kaplan-Meier method and compared between the groups using the Log rank test. A two-tailed $P$ value of $<0.05$ was considered statistically significant.

\section{Results \\ Expression of VCAM-I in NPC}

To determine the role of VCAM-1 in the prognosis of NPC, 73 patients diagnosed with NPC were included in the study. Immunohistochemical analysis showed that VCAM-1 protein is mainly expressed in the cytoplasm, cell membrane, and partly in the nucleus in NPC tissues, which were stained brown and showed no expression in inflammatory tissues (Figure 1). The staining MOD values of VCAM-1 in NPC and chronic inflammatory tissues were $0.1946 \pm 0.0415$ and $0.0944 \pm 0.0245$, respectively. The expression of VCAM-1 protein in NPC was significantly higher than that in chronic inflammatory tissue $(P=0.003)$ (Figure 2). For further analysis, 73 patients with NPC were divided into two groups: low expression group $(n=32)$ and high expression group $(n=41)$ based on the median value $(0.1888)$ of MOD.

\section{Relationship Between VCAM-I Expression and Clinicopathological Factors in NPC}

The demographic and clinical characteristics of the 73 patients with NPC are shown in Table 1. No significant differences in the expression of VCAM-1 among gender, 

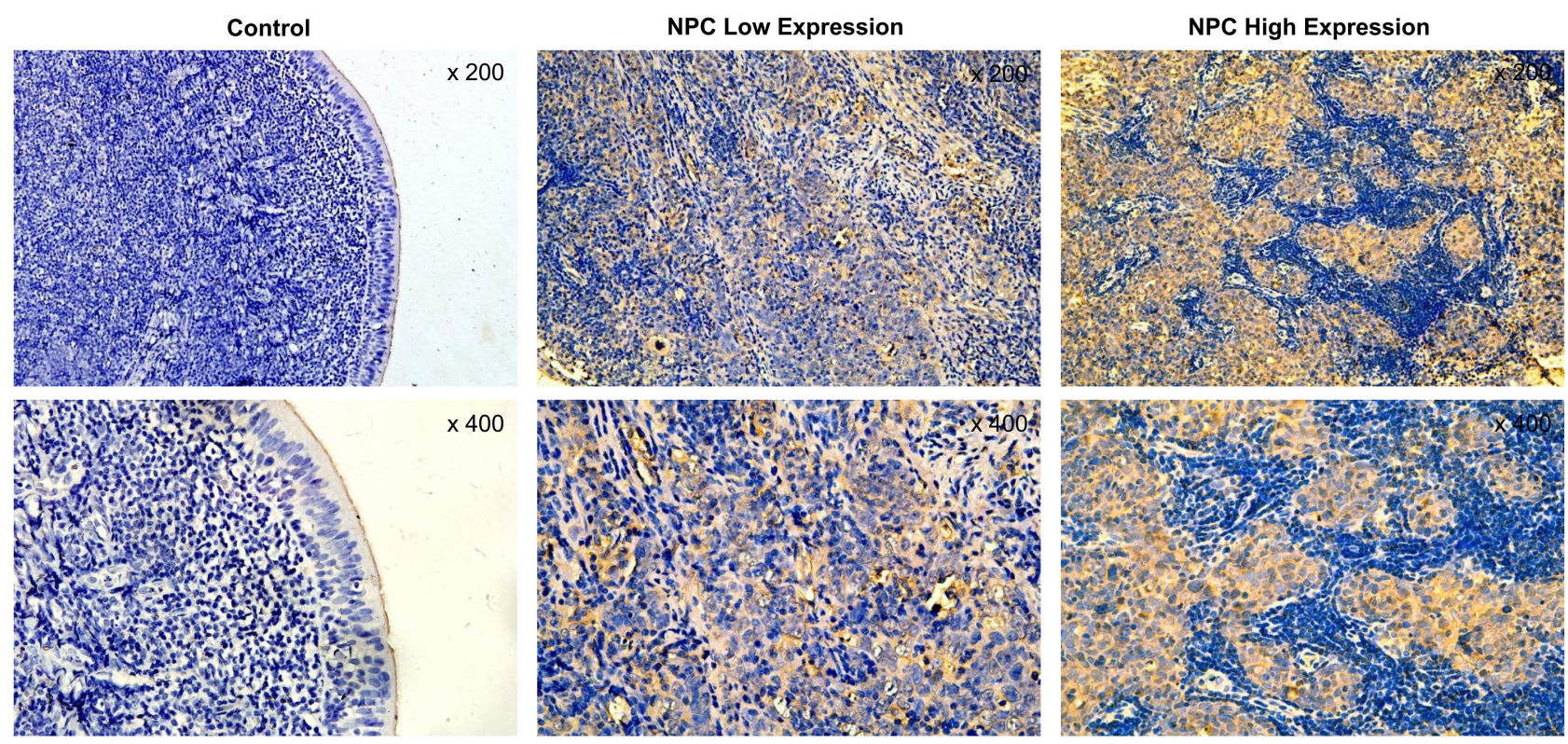

Figure I The expression of VCAM-I in chronic inflammatory and NPC tissues. VCAM-I is mainly expressed in the cytoplasm, cell membrane, and partly in the nucleus in NPC tissues.

age, pathologic classification, tumor classification, lymph node status, metastasis status, and overall clinical stage were found (all $P>0.05$ ) (Table 1).

\section{Prognostic Value of VCAM-I Expression}

Stratification analyses indicated that the tumor progression rate of NPC patients in the VCAM-1 high expression group (46.34\%) was not statistically different from that in the low expression group (31.25\%) $(P=0.191)$. However, there was a significant difference in the rates of mortality between these

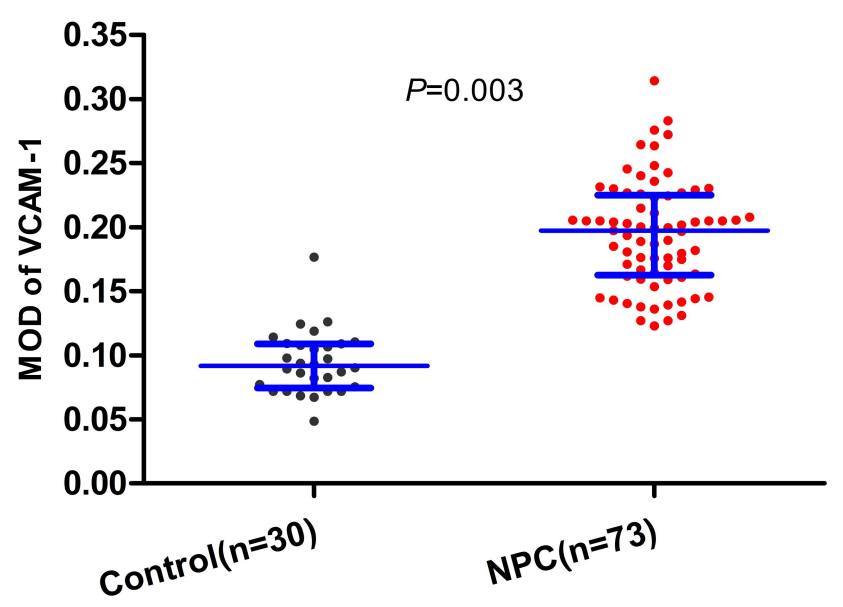

Figure 2 Levels of VCAM-I expression in inflammatory controls and patients with NPC. The expression of VCAM-I in NPC $(0.1946 \pm 0.0415)$ was significantly higher than that in chronic inflammatory tissue $(0.0944 \pm 0.0245)$. two groups of patients $(34.15 \%$ vs $12.50 \%$, respectively, $P=0.033$ ) (Figure $3 \mathrm{~A}$ and $\mathrm{B}$ ).

Moreover, log-rank analyses revealed that there was a significant difference in the periods of PFS and OS between these two groups of patients $(P=0.044$ and $P=0.024$, respectively). The periods of PFS and OS in patients with high VCAM-1 expression were significantly shorter than those in patients with low VCAM-1 expression (Figure 3C and D).

\section{Association of ATP-TCA Results with VCAM-I Expression}

Because of the small sample size, the results of tumor chemosensitivity in this study were sorted into two types (resistance and sensitivity) for further analysis. The sensitivity of NPC to eight chemotherapy drugs was different $(P<0.001)$. The sensitivity rates of the chemotherapy drugs were as follows: fluorouracil, $82.19 \%$; cisplatin, $89.04 \%$; carboplatin, 93.15\%; oxaliplatin, 91.78\%; paclitaxel, 79.45\%; docetaxel, 61.64\%; gemcitabine, 64.38\%; and cyclophosphamide, $75.34 \%$. Carboplatin showed the highest sensitivity rate, and docetaxel showed the highest resistance rate (Figure 4A and B).

Further analyses showed that the resistance rate of NPC with high expression of VCAM-1 to carboplatin was over three times that of those with low expression of VCAM-1 $(9.76 \%$ vs $3.13 \%, P=0.266)$. However, the resistance rate of NPC with low expression of VCAM-1 to 
Table I Clinical Characteristics and VCAM-I Expression in Patients with NPC ( $n=73)$

\begin{tabular}{|c|c|c|c|c|}
\hline \multicolumn{2}{|l|}{ Characteristics } & \multirow{2}{*}{$\begin{array}{r}\text { No. (\%) } \\
49(67.12 \%) \\
24(32.88 \%)\end{array}$} & \multirow{2}{*}{$\begin{array}{c}\text { MOD Value } \\
0.1945 \pm 0.0437 \\
0.1948 \pm 0.0374\end{array}$} & \multirow{2}{*}{$\begin{array}{r}P \\
0.265\end{array}$} \\
\hline Gender & $\begin{array}{l}\text { Male } \\
\text { Female }\end{array}$ & & & \\
\hline Age, years & $\begin{array}{l}<48 \\
\geq 48\end{array}$ & $\begin{array}{l}33(45.21 \%) \\
40(54.79 \%)\end{array}$ & $\begin{array}{l}0.1885 \pm 0.0395 \\
0.1996 \pm 0.0429\end{array}$ & 0.533 \\
\hline WHO pathologic type ${ }^{a}$ & $\begin{array}{l}\text { Keratinizing carcinoma } \\
\text { Differentiated non-keratinizing carcinoma } \\
\text { Undifferentiated non-keratinizing carcinoma }\end{array}$ & $\begin{array}{r}3(4.11 \%) \\
34(46.57 \%) \\
36(49.32 \%)\end{array}$ & $\begin{array}{l}0.2040 \pm 0.0685 \\
0.1947 \pm 0.0425 \\
0.1936 \pm 0.0395\end{array}$ & 0.919 \\
\hline Tumor classification $^{\mathbf{b}}$ & $\begin{array}{l}\mathrm{T}_{1} \\
\mathrm{~T}_{2} \\
\mathrm{~T}_{3} \\
\mathrm{~T}_{4}\end{array}$ & $\begin{array}{r}11(15.07 \%) \\
26(35.62 \%) \\
27(36.98 \%) \\
9(12.33 \%)\end{array}$ & $\begin{array}{l}0.2018 \pm 0.0430 \\
0.1865 \pm 0.0410 \\
0.1990 \pm 0.0448 \\
0.1958 \pm 0.0329\end{array}$ & 0.662 \\
\hline Lymph node status ${ }^{\mathrm{b}}$ & $\begin{array}{l}N_{0} \\
N_{1} \\
N_{2} \\
N_{3}\end{array}$ & $\begin{array}{r}16(21.92 \%) \\
14(19.18 \%) \\
37(50.68 \%) \\
6(8.22 \%)\end{array}$ & $\begin{array}{l}0.1886 \pm 0.0427 \\
0.1935 \pm 0.0365 \\
0.1942 \pm 0.0412 \\
0.2153 \pm 0.0548\end{array}$ & 0.614 \\
\hline Metastasis status ${ }^{\mathbf{b}}$ & $\begin{array}{l}M_{0} \\
M_{1}\end{array}$ & $\begin{array}{r}69(94.52 \%) \\
4(5.48 \%)\end{array}$ & $\begin{array}{l}0.194 I \pm 0.0423 \\
0.2026 \pm 0.0250\end{array}$ & 0.693 \\
\hline Overall clinical stage $^{b}$ & $\begin{array}{l}\text { I } \\
\text { II } \\
\text { III } \\
\text { IV }\end{array}$ & $\begin{array}{r}3(4.11 \%) \\
10(13.70 \%) \\
45(61.64 \%) \\
15(20.55 \%)\end{array}$ & $\begin{array}{l}0.2091 \pm 0.0294 \\
0.2094 \pm 0.044 \mid \\
0.1887 \pm 0.0414 \\
0.1995 \pm 0.0418\end{array}$ & 0.436 \\
\hline
\end{tabular}

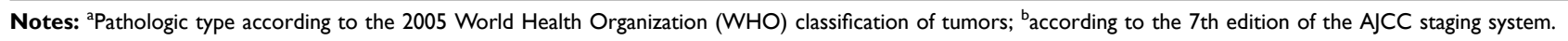

paclitaxel was over three times that of those with high expression of VCAM-1 (34.38\% vs $9.76 \%, P=0.010)$. There were no obvious differences in the chemotherapeutic drug resistance rates of the other six drugs between the two groups (Figure 4C).

\section{Discussion}

Cell adhesion molecules are proteins located on the cell surface, which are involved in the process of cellular adhesion and binding with other cells or the extracellular matrix. ${ }^{26}$ Most cell adhesion molecules belong to four protein families, the immunoglobulin superfamily, integrin, calcine, and selectin. ${ }^{27}$ VCAM-1 is a member of the immunoglobulin superfamily, which plays an important role in diseases such as inflammation, ${ }^{28}$ rheumatoid arthritis, ${ }^{29}$ asthma, ${ }^{30}$ and transplant rejection. ${ }^{31}$ Numerous studies have confirmed that VCAM-1 is highly expressed in breast cancer, ${ }^{13,14}$ gastric cancer, ${ }^{15}$ colorectal cancer, ${ }^{16}$ lung cancer, ${ }^{17}$ ovarian cancer, ${ }^{18}$ head and neck cancers, ${ }^{23}$ and could lead to a poor prognosis. VCAM-1 is related to tumor angiogenesis, metastasis, and resistance to chemotherapy drugs, ${ }^{20-22,24}$ and is considered a potential target for tumor anti-vascular and anti-metastatic therapies. ${ }^{19}$ However, there are few reports on VCAM-1 expression in NPC and its association with chemotherapy resistance and prognosis.

In this study, the expression of VCAM-1 in NPC tissue was significantly higher than that in chronic inflammatory nasopharyngeal tissue, while the expression of VCAM-1 did not differ among gender, age, pathologic classification, tumor classification, lymph node status, metastasis status, and overall clinical stage, which is consistent with a previous report. ${ }^{23}$ Survival analyses revealed that the periods of PFS and OS in patients with high VCAM-1 expression were significantly shorter than those in patients with low VCAM-1 expression. VCAM-1 could be a prognostic marker of NPC patients. This indicates that VCAM-1 is universally expressed in NPC and may play an important role in the initial development and treatment of NPC.

Radiotherapy combined with chemotherapy could increase the five-year survival rate of locally advanced NPC by $6 \%$ and the event-free survival rate by $10 \% .{ }^{32}$ On the other hand, concurrent radiotherapy and chemotherapy have been proven to be the standard treatment 

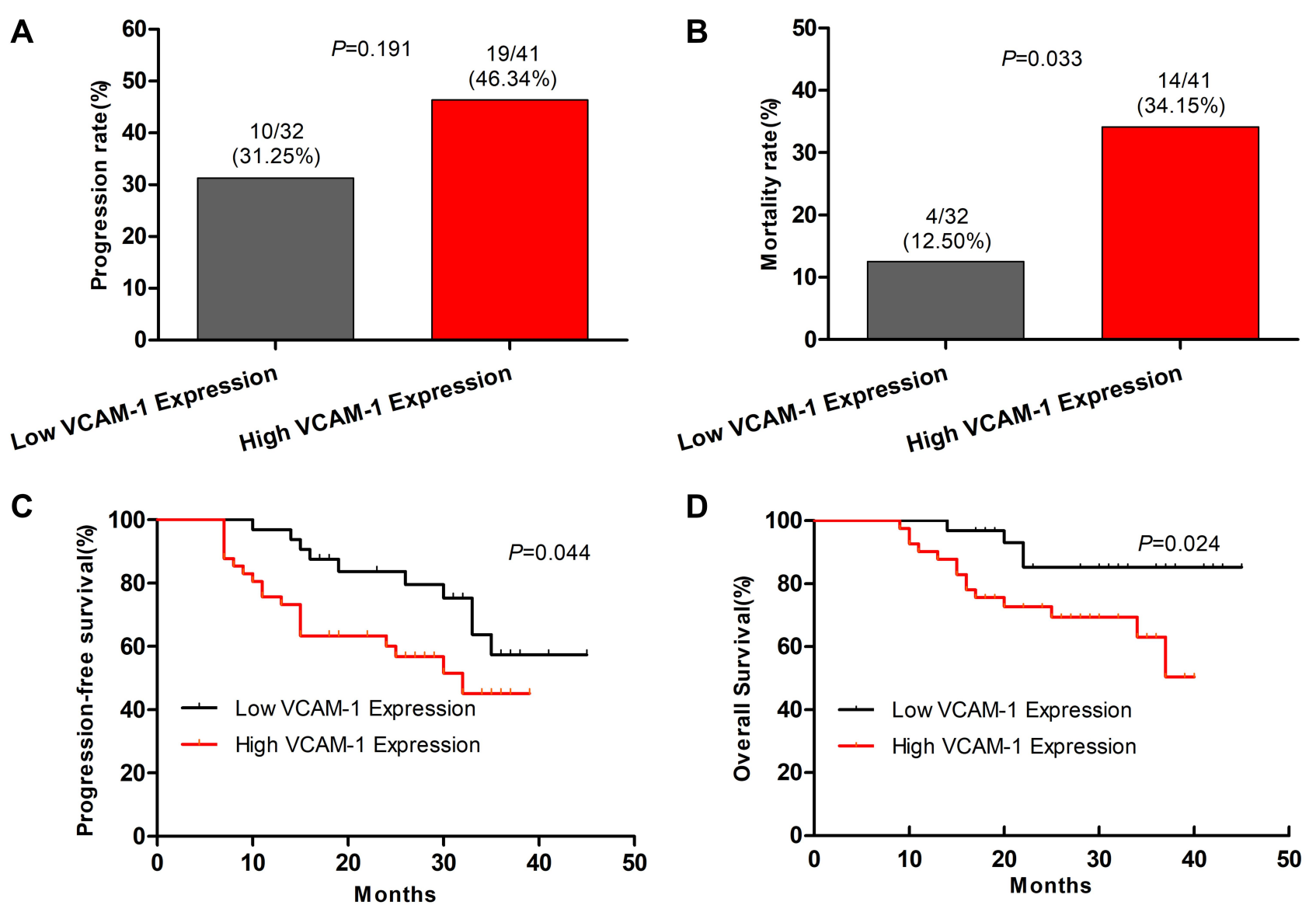

Figure 3 Stratification analyses of the survival of patients with NPC. All of the NPC patients were stratified according to VCAM-I level, and their NPC relapse and mortality were calculated. Subsequently, the RFS and OS periods of individual groups of patients were estimated by the Kaplan-Meier method and analyzed by the Log rank test. (A) The percentages of progression. (B) The percentages of mortality. (C) The percentages of PFS in different groups of patients with NPC throughout the observation period. (D) The percentages of OS in the different groups of patients with NPC throughout the observation period.

modalities for locally advanced NPC, which significantly reduces the risk of local recurrence and distant metastasis. ${ }^{2}$ Induction chemotherapy has received increasing attention in recent years because it can reduce tumor burden, improve radiotherapy sensitivity, eliminate or inhibit micrometastases, and reduce clinical symptoms caused by tumors in a short time. ${ }^{33}$ Common drug combinations administered in this study were TP (paclitaxel + cisplatin), FP (fluorouracil + cisplatin), TPF (paclitaxel + fluorouracil + cisplatin), and GP (gemcitabine + cisplatin). The ATPTCA results of 73 fresh NPC tissues showed that NPC cells are generally sensitive to fluorouracil, cisplatin, carboplatin, oxaliplatin, paclitaxel, docetaxel, gemcitabine, and cyclophosphamide, which are commonly used as clinical chemotherapy drugs for NPC. However, the sensitivity rates of NPC to fluorouracil and platinum-based drugs were higher than those to paclitaxel, docetaxel, gemcitabine, and cyclophosphamide. This may also explain why fluorouracil and platinum-based chemotherapeutics are often selected as the main therapeutic drug combination. In this study, the single-drug resistance rate of NPC to gemcitabine was significantly higher than that to fluorouracil. However, it was confirmed that the GP regimen is superior to the FP regimen in terms of efficiency, PFS, and $\mathrm{OS},{ }^{34}$ which may be related to the mutual sensitization of chemotherapy drugs.

At the same time, VCAM-1 was found to be involved in chemotherapy resistance. Inhibition of VCAM-1 expression in acute myeloid leukemia (AML) enhances the sensitivity to chemotherapy drugs. ${ }^{22,35}$ VCAM-1, as a downstream target gene of Interleukin-8, participated in the resistance of gastric cancer cells to oxaliplatin. ${ }^{20}$ In breast cancer cells, overexpression of VCAM-1 could lead to chemoresistance to doxorubicin and cisplatin, which is related to TGF $\beta 1$, IL6 , and epithelial and mesenchymal transition (EMT). ${ }^{21}$ In our study, the resistance rate of NPC with low expression of VCAM-1 to paclitaxel was over three times that of those with high expression of VCAM-1 $(P=0.010)$. Although the 

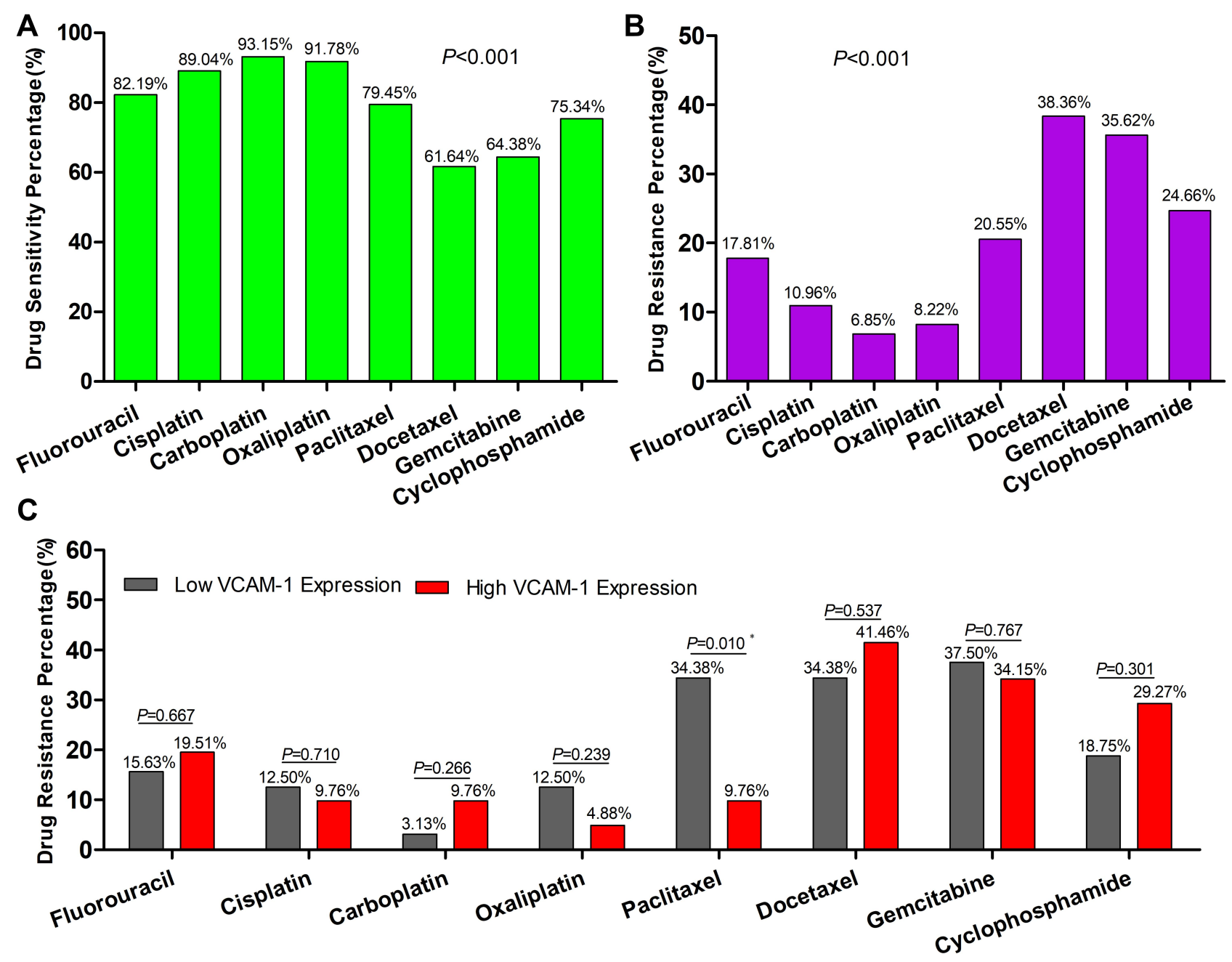

Figure 4 The analyses of resistance to eight chemotherapy drugs in patients with NPC. All of the NPC patients were stratified according to VCAM-I level, and their resistances to eight chemotherapy drugs were analyzed. (A) The percentages of drug sensitivity. (B) The percentages of drug resistance. (C) The percentages of drug resistance in the different groups of patients with NPC.

single-drug resistance rate of NPC to paclitaxel was $20.55 \%$, paclitaxel could still be chosen as a chemotherapy drug for patients with high expression of VCAM-1 in clinical practice. The resistance rate of NPC with high expression of VCAM-1 to carboplatin was over three times that of those with low expression of VCAM-1 $(P=0.266)$, which needs further confirmation through future studies with larger sample sizes. Carboplatin was the drug that showed the highest chemotherapy sensitivity rate (93.15\%). However, carboplatin might not be the preferred platinum chemotherapy drug for patients with high expression of VCAM-1. This result suggested that VCAM-1 might be the target of chemotherapy drug selection for a more accurate evaluation of carboplatin and paclitaxel, which are first-line clinical chemotherapy drugs for NPC. VCAM-1 was found to be highly related to angiogenesis in NPC. ${ }^{24}$ Targeting the perivascular VCAM-1 as one of the multiple endothelialderived integrin $\alpha 4 \beta 1$ ligands sensitized breast tumor cells to chemotherapy. ${ }^{36,37}$ VCAM-1 may affect chemotherapy resistance by enhancing angiogenesis in NPC. However, the specific mechanism requires further study.

Our study had some limitations as well. First, the population enrolled in the study was considerably small. Second, we cannot perform a subgroup analysis of the relationship between tumor chemosensitivity and clinicopathological factors owing to the small sample size. Moreover, all NPC patients originated from one center and there was no validation cohort. Therefore, larger sample-sized, prospective, multicenter, randomized, and controlled clinical studies are required to further validate our findings. Also, NPC cells will be used to study the molecular mechanism of VCAM-1 affecting the sensitivity of chemotherapy drugs. 


\section{Conclusion}

In summary, our data indicated that VCAM-1 was highly expressed in NPC. Patients with high VCAM-1 expression were more prone to shorter periods of PFS and OS. VCAM-1 could be a prognostic marker of NPC patients. The detection of VCAM-1 expression in NPC may be valuable for evaluating and selecting carboplatin and paclitaxel in the clinic. Therefore, our findings may provide a new reference for chemotherapy drug evaluation and management of patients with NPC.

\section{Data Sharing Statement}

The data used to support the findings of this study are restricted by the Ethics Committee of the Affiliated Hospital of Southwest Medical University to protect patient privacy. Data are available from the corresponding authors upon request for researchers who meet the criteria for access to confidential data.

\section{Acknowledgments}

This work was supported by the National Natural Science Foundation of China under Grant [81602570]; the Health Commission of Sichuan Province under Grant [17ZB0465]; and the Education Department of Sichuan Province under Grant [17PJ532]. Language help was provided by Elsevier Webshop language services.

\section{Disclosure}

The authors report no conflicts of interest in this work.

\section{References}

1. Chua MLK, Wee JTS, Hui EP, Chan ATC. Nasopharyngeal carcinoma. Lancet. 2016;387(10022):1012-1024. doi:10.1016/S0140-6736(15) 00055-0

2. Colevas AD, Yom SS, Pfister DG, et al. NCCN guidelines insights: head and neck cancers, version 1.2018. J Nat Compr Cancer Network. 2018;16(5):479-490. doi:10.6004/jncen.2018.0026

3. Chen YP, Chan ATC, Le QT, et al. Nasopharyngeal carcinoma. Lancet. 2019;394(10192):64-80. doi:10.1016/S0140-6736(19)30956-0

4. Bissey PA, Teng M, Law JH, et al. MiR-34c downregulation leads to SOX4 overexpression and cisplatin resistance in nasopharyngeal carcinoma. BMC Cancer. 2020;20(1):597. doi:10.1186/s12885-02007081-z

5. Zhang P, Lu X, Shi $Z$, et al. miR-205-5p regulates epithelial-mesenchymal transition by targeting PTEN via PI3K/AKT signaling pathway in cisplatin-resistant nasopharyngeal carcinoma cells. Gene. 2019;710:103-113. doi:10.1016/j.gene.2019.05.058

6. Zhang R, Li SW, Liu L, et al. TRIM11 facilitates chemoresistance in nasopharyngeal carcinoma by activating the $\beta$-catenin/ABCC9 axis via p62-selective autophagic degradation of Daple. Oncogenesis. 2020;9 (5):45. doi:10.1038/s41389-020-0229-9
7. Wei X, Nian J, Zheng J, et al. Inhibition of cyclin-dependent kinases by AT7519 enhances nasopharyngeal carcinoma cell response to chemotherapy. Cancer Chemother Pharmacol. 2020;85(5):949-957. doi:10.1007/s00280-020-04068-2

8. Zhang J, Xie T, Zhong X, et al. Melatonin reverses nasopharyngeal carcinoma cisplatin chemoresistance by inhibiting the Wnt $/ \beta$-catenin signaling pathway. Aging. 2020;12(6):5423-5438. doi:10.18632/ aging. 102968

9. Rice GE, Bevilacqua MP. An inducible endothelial cell surface glycoprotein mediates melanoma adhesion. Science. 1989;246 (4935):1303-1306. doi:10.1126/science.2588007

10. Cook-Mills JM, Marchese ME, Abdala-Valencia H. Vascular cell adhesion molecule-1 expression and signaling during disease: regulation by reactive oxygen species and antioxidants. Antioxid Redox Signal. 2011;15(6):1607-1638. doi:10.1089/ars.2010.3522

11. Sharma R, Khaket TP, Dutta C, et al. Breast cancer metastasis: putative therapeutic role of vascular cell adhesion molecule-1. Cell Oncol. 2017;40(3):199-208. doi:10.1007/s13402-017-0324-x

12. Kong DH, Kim YK, Kim MR, et al. Emerging roles of Vascular Cell Adhesion Molecule-1 (VCAM-1) in immunological disorders and cancer. Int J Mol Sci. 2018;19:4. doi:10.3390/ijms 19041057

13. Minn AJ, Gupta GP, Siegel PM, et al. Genes that mediate breast cancer metastasis to lung. Nature. 2005;436(7050):518-524. doi:10.1038/nature03799

14. Byrne GJ, Ghellal A, Iddon J, et al. Serum soluble vascular cell adhesion molecule-1: role as a surrogate marker of angiogenesis. J Natl Cancer Inst. 2000;92(16):1329-1336. doi:10.1093/jnci/92.16.1329

15. Ding YB, Chen GY, Xia JG, et al. Association of VCAM-1 overexpression with oncogenesis, tumor angiogenesis and metastasis of gastric carcinoma. World J Gastroenterol. 2003;9(7):1409-1414. doi:10.3748/wjg.v9.i7.1409

16. Zhang R, Qi F, Zhao F, et al. Cancer-associated fibroblasts enhance tumor-associated macrophages enrichment and suppress NK cells function in colorectal cancer. Cell Death Dis. 2019;10(4):273. doi:10.1038/s41419-019-1435-2

17. Kim MR, Jang JH, Park CS, et al. A human antibody that binds to the sixth Ig-like domain of VCAM-1 blocks lung cancer cell migration in vitro. Int J Mol Sci. 2017;18:3.

18. Scalici JM, Arapovic S, Saks EJ, et al. Mesothelium expression of vascular cell adhesion molecule-1 (VCAM-1) is associated with an unfavorable prognosis in epithelial ovarian cancer (EOC). Cancer. 2017;123(6):977-984. doi:10.1002/cncr.30415

19. Kim TK, Park CS, Na HJ, et al. Ig-like domain 6 of VCAM-1 is a potential therapeutic target in TNF $\alpha$-induced angiogenesis. Exp Mol Med. 2017;49(2):e294. doi:10.1038/emm.2016.147

20. Kuai WX, Wang Q, Yang XZ, et al. Interleukin-8 associates with adhesion, migration, invasion and chemosensitivity of human gastric cancer cells. World $J$ Gastroenterol. 2012;18(9):979-985. doi:10.3748/wjg.v18.i9.979

21. Wang PC, Weng CC, Hou YS, et al. Activation of VCAM-1 and its associated molecule CD44 leads to increased malignant potential of breast cancer cells. Int $J$ Mol Sci. 2014;15(3):3560-3579. doi:10.3390/ijms15033560

22. Becker PS, Kopecky KJ, Wilks AN, et al. Very late antigen-4 function of myeloblasts correlates with improved overall survival for patients with acute myeloid leukemia. Blood. 2009;113(4):866-874. doi:10.1182/blood-2007-12-124818

23. Liu CM, Sheen TS, Ko JY, Shun CT. Circulating intercellular adhesion molecule 1 (ICAM-1), E-selectin and vascular cell adhesion molecule 1 (VCAM-1) in head and neck cancer. $\mathrm{Br} J$ Cancer. 1999;79(2):360-362. doi:10.1038/sj.bjc.6690057

24. Cheng S, Li Z, He J, et al. Epstein-Barr virus noncoding RNAs from the extracellular vesicles of nasopharyngeal carcinoma (NPC) cells promote angiogenesis via TLR3/RIG-I-mediated VCAM-1 expression. Biochimica et biophysica acta. Mol Basis Dis. 2019;1865 (6):1201-1213. doi:10.1016/j.bbadis.2019.01.015 
25. Kurbacher CM, Cree IA, Bruckner HW, et al. Use of an ex vivo ATP luminescence assay to direct chemotherapy for recurrent ovarian cancer. Anticancer Drugs. 1998;9(1):51-57. doi:10.1097/00001813199801000-00006

26. Zou R, Shi W, Tao J, et al. SIRT5 and post-translational protein modifications: a potential therapeutic target for myocardial ischemia-reperfusion injury with regard to mitochondrial dynamics and oxidative metabolism. Eur J Pharmacol. 2018;818:410-418. doi:10.1016/j.ejphar.2017.11.005

27. Nan J, Zhu W, Rahman MS, et al. Molecular regulation of mitochondrial dynamics in cardiac disease. Biochim Biophys Acta Mol Cell Res. 2017;1864(7):1260-1273. doi:10.1016/j.bbamcr.2017.03.006

28. Cerutti C, Ridley AJ. Endothelial cell-cell adhesion and signaling. Exp Cell Res. 2017;358(1):31-38. doi:10.1016/j.yexcr.2017.06.003

29. Wang L, Ding Y, Guo X, Zhao Q. Role and mechanism of vascular cell adhesion molecule-1 in the development of rheumatoid arthritis. Exp Ther Med. 2015;10(3):1229-1233. doi:10.3892/etm.2015.2635

30. Steinke JW, Borish L. Th2 cytokines and asthma. Interleukin-4: its role in the pathogenesis of asthma, and targeting it for asthma treatment with interleukin-4 receptor antagonists. Respir Res. 2001;2 (2):66-70. doi:10.1186/rr40

31. Piotti G, Palmisano A, Maggiore U, Buzio C. Vascular endothelium as a target of immune response in renal transplant rejection. Front Immunol. 2014;5:505. doi:10.3389/fimmu.2014.00505
32. Baujat B, Audry H, Bourhis J, et al. Chemotherapy in locally advanced nasopharyngeal carcinoma: an individual patient data meta-analysis of eight randomized trials and 1753 patients. Int J Radiat Oncol Biol Phys. 2006;64(1):47-56. doi:10.1016/j. ijrobp.2005.06.037

33. Blanchard P, Lee A, Marguet S, et al. Chemotherapy and radiotherapy in nasopharyngeal carcinoma: an update of the MAC-NPC meta-analysis. Lancet Oncol. 2015;16(6):645-655. doi:10.1016/ S1470-2045(15)70126-9

34. Zhang L, Huang Y, Hong S, et al. Gemcitabine plus cisplatin versus fluorouracil plus cisplatin in recurrent or metastatic nasopharyngeal carcinoma: a multicentre, randomised, open-label, Phase 3 trial. Lancet. 2016;388(10054):1883-1892. doi:10.1016/S0140-6736(16)31388-5

35. Bosse RC, Wasserstrom B, Meacham A, et al. Chemosensitizing AML cells by targeting bone marrow endothelial cells. Exp Hematol. 2016;44(5):363-377 e365. doi:10.1016/j.exphem.2016.02.003

36. Garmy-Susini B, Jin H, Zhu Y, et al. Integrin alpha4beta1-VCAM1 -mediated adhesion between endothelial and mural cells is required for blood vessel maturation. J Clin Invest. 2005;115(6):1542-1551. doi: $10.1172 / \mathrm{JCI} 23445$

37. Carlson P, Dasgupta A, Grzelak CA, et al. Targeting the perivascular niche sensitizes disseminated tumor cells to chemotherapy. Nat Cell Biol. 2019;21(2):238-250. doi:10.1038/s41556-018-0267-0
OncoTargets and Therapy

\section{Publish your work in this journal}

OncoTargets and Therapy is an international, peer-reviewed, open access journal focusing on the pathological basis of all cancers, potential targets for therapy and treatment protocols employed to improve the management of cancer patients. The journal also focuses on the impact of management programs and new therapeutic

\section{Dovepress}

agents and protocols on patient perspectives such as quality of life, adherence and satisfaction. The manuscript management system is completely online and includes a very quick and fair peer-review system, which is all easy to use. Visit http://www.dovepress.com/ testimonials.php to read real quotes from published authors. 